Proc. XII Int. School on Theoretical Physics — Symmetry and Structural Properties of Condensed Matter

\title{
Anisotropy of $g_{\text {eff }}$ Factor for $\mathrm{Ni}_{50} \mathrm{Mn}_{35.5} \mathrm{In}_{14.5}$ Heusler Alloy
}

\author{
Ł. DUBIEL ${ }^{a, b, *}$, A. WAL ${ }^{c}$ AND I. STEFANIUK ${ }^{d}$
}

${ }^{a}$ Faculty of Mathematics and Natural Sciences, Department of Biophysics, University of Rzeszów, S. Pigonia 1, 35-959 Rzeszów, Poland

${ }^{b}$ International PhD Studies, Institute of Nuclear Physics, Polish Academy of Sciences,

E. Radzikowskiego 152, 31-342 Kraków, Poland

${ }^{c}$ Faculty of Mathematics and Natural Sciences, Department of Theoretical Physics, University of Rzeszów,

S. Pigonia 1, 35-959 Rzeszów, Poland

${ }^{d}$ Faculty of Mathematics and Natural Sciences, Center for Microelectronics and Nanotechnology,

University of Rzeszów, S. Pigonia 1, 35-959 Rzeszów, Poland

In this work electron magnetic resonance (EMR) spectra of off-stoichiometric $\mathrm{Ni}_{50} \mathrm{Mn}_{35.5} \mathrm{In}_{14.5}$ Heusler alloy ribbons obtained by melt-spinning method were measured. The EMR measurements were performed on the Xband $(9.36 \mathrm{GHz})$ spectrometer. Angle dependences were registered for three orientations of sample, with the use of uniaxial goniometer at 300 . The results exhibited magnetic anisotropy and were used for calculating the principal values of $g$ tensor.

DOI: 10.12693/APhysPolA.132.91

PACS/topics: 76.30.-v, 75.20.En, 32.10.Fn

\section{Introduction}

The Heusler alloys Ni-Mn-Z $(\mathrm{Z}=\mathrm{Ga}, \mathrm{Sb}, \mathrm{Sn}, \mathrm{In})$ have become an attractive group of materials due to their properties: semimetallic, large magnetoresistance (MR), giant magnetocaloric effect (giant $\mathrm{MCE}$ ), ferromagnetic shape memory effect (FSME), anisotropy exchange bias (EB) [1-6]. They also have potential practical use in many different fields e.g. spintronics, ecological refrigeration. The properties of the Heusler alloys could be changed by the stoichiometry, temperature, and magnetic field variation $[4,6]$.

The Heusler alloys have been divided due to the crystal structure into two groups [7]. The first is the fullHeusler alloys, with a general formula $\mathrm{X}_{2} \mathrm{YZ}$, which crystallize in the $L 2_{1}$ structure. $L 2_{1}$ structure can be described by means of four interpenetrating fcc sublattices: two sublattices for $\mathrm{X}$ atoms can be characterized by positions $(0,0,0),\left(\frac{1}{2}, \frac{1}{2}, \frac{1}{2}\right)$ and $\mathrm{Y}$ by $\left(\frac{3}{4}, \frac{3}{4}, \frac{3}{4}\right)$ and $\mathrm{Z}$ by $\left(\frac{1}{4}, \frac{1}{4}, \frac{1}{4}\right)[1]$. The second type of the Heusler alloys family is half-Heusler alloys with composition XYZ, which crystallize in $C 1_{b}$ structure. This structure has three interpenetrating fcc lattice and is similar to the $L 2_{1}$ structure, but one $\mathrm{X}$ sublattice $\left(\frac{1}{2}, \frac{1}{2}, \frac{1}{2}\right)$ is vacant, Fig. 1 .

The majority of contemporary work on magnetic Heusler alloys is related to stoichiometric Heusler system. From the other hand, recent significant research has been focused on the magnetic, structural, and thermodynamic properties of alloys that have off-stoichiometric form. The $\mathrm{Ni}_{50} \mathrm{Mn}_{25+x} \mathrm{Z}_{25-x}$ alloys $(\mathrm{Z}=\mathrm{Ga}, \mathrm{Sb}, \mathrm{Sn}, \mathrm{In}$,

*corresponding author; e-mail: lukasz.dubiel@ifj.edu.pl etc.) is derived from stoichiometric full Heusler $\mathrm{Ni}_{2} \mathrm{Mn}-$ $\mathrm{Z}[1,2]$ by deliberate substitution of $\mathrm{Mn}$ on the $\mathrm{Z}$ site. Recently, Ni-Mn-In and $\mathrm{Ni}-\mathrm{Co}-\mathrm{Mn}-\mathrm{In}$ Heusler alloys have attracted much attention due to their potential application in ecological refrigeration.

In this research, we registered electron magnetic resonance (EMR) spectra of a $\mathrm{Ni}_{50} \mathrm{Mn}_{35.5} \operatorname{In}_{14.5}$ ribbons [8] which were obtained by melt-spinning method. Based on resonance field $B_{\text {res }}$ measurements for different sample orientation in relation to static field, we calculated the principal values of $g_{\text {eff }}$ tensor.

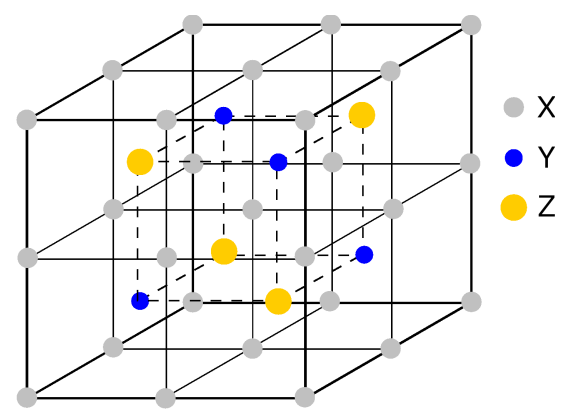

Fig. 1. Crystal structure of $\mathrm{X}_{2} \mathrm{YZ}$ in the $L 2_{1}$.

\section{Experimental}

Samples were prepared by melt-spinning method [8]. The EMR measurements were performed on Bruker ELEXSYS E580 multifrequency spectrometer using the $\mathrm{X}$-band $(9.36 \mathrm{GHz})$. Angular dependences (with a 10 degrees step) were registered in three orientation of sample with the use of single-axis goniometer. Each rotation axis was perpendicular to planes of sample (see Fig. 2). 


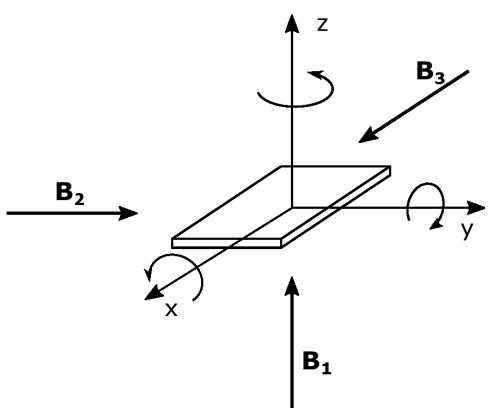

Fig. 2. Orientation of sample with respect to static field $\boldsymbol{B}_{i} . \boldsymbol{B}_{i}$ denotes the direction of magnetic field for succeeding measurements.

Spectra were registered at 300 K. Before measurements we applied special procedures, because previous research shows time influence on signal shape and saturation of line (i.e. repetition of measurement changes shape of signal and the $B_{\text {res }}$ value). First sample was gradually heated to $400 \mathrm{~K}$ and then instantly cooled to $300 \mathrm{~K}$.

\section{Results and discussion}

The EMR spectra of the investigated sample at $300 \mathrm{~K}$ for three perpendicular orientations are shown in Fig. 3. For rotation around the $x$ - and $y$-axis, the spectra exhibited angular dependence. The rotation around $z$-axis did not reveal any changes of lines. The obtained spectra contain single, strong, asymmetric lines. The asymmetry EMR line suggested that investigated sample is conductive and could be described by the Dyson model $[9,10]$.

The EMR spectra of $\mathrm{Ni}_{50} \mathrm{Mn}_{35.5} \operatorname{In}_{14.5}$ ribbons were satisfactory fitted by derivative of Dyson function [10]:

$$
\begin{gathered}
P(B)=\frac{\Delta B+\alpha\left(B-B_{\text {res }}\right)}{4\left(B-B_{\text {res }}\right)^{2}} \\
+\frac{\Delta B+\alpha\left(B+B_{\text {res }}\right)}{4\left(B+B_{\text {res }}\right)^{2}+B_{\text {res }}{ }^{2}},
\end{gathered}
$$

where $B_{r e s}$ is the resonance field, $\Delta B$ is the line width and $\alpha$ is parameter of asymmetry which represents relation between absorption and dispersion. The Dyson function $P(B)$ is related to power absorption, but in practice EMR signals are recorded as derivatives of $P(B)$ [10]. The sample signals EMR of $\mathrm{Ni}_{50} \mathrm{Mn}_{35.5} \operatorname{In}_{14.5}$ and their fittings are presented in Fig. 4.

The angular dependences of the resonance field of the sample in three positions (Fig. 2), obtained from the Dyson fitting are shown in Fig. 5. The dependences of the resonance field have different character for each orientation. In the case where sample was rotated around $x$ - or $y$-axis, resonance field changed in relatively broad range - from 3150 Gs to 3460 Gs for rotation around $x$ axis and from 3140 to 3510 Gs for rotation around $y$-axis. The dependences for $z$-axis did not reveal any changes of $B_{\text {res }}$ values.

The resonance field values were used to calculate effective $g$-factor [11]:
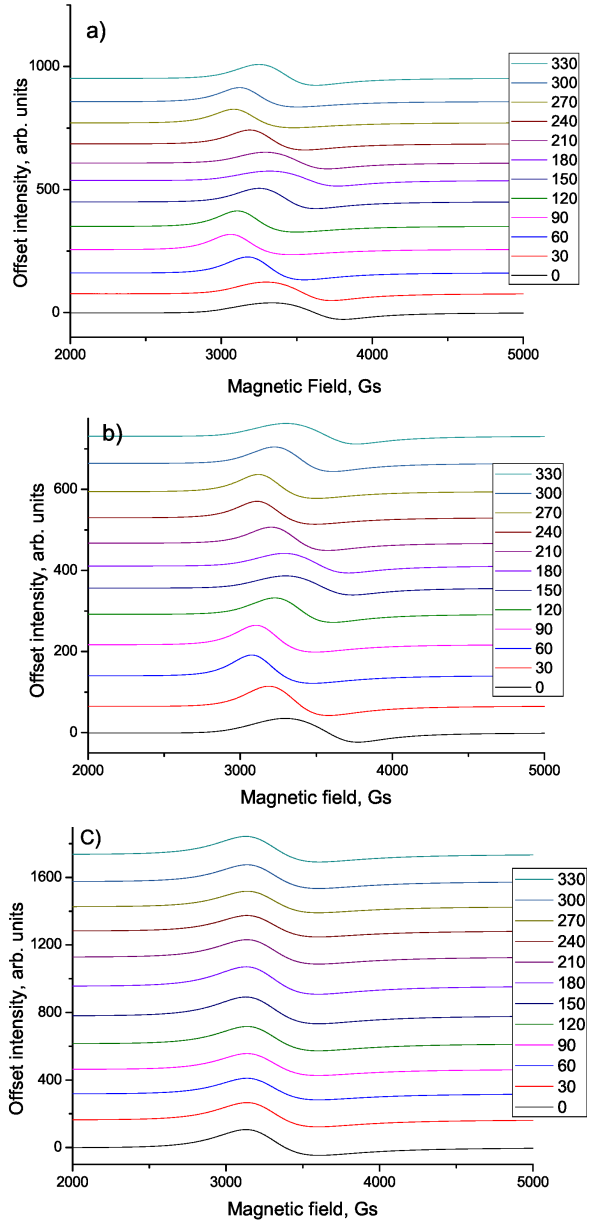

Fig. 3. The angular dependences of $\mathrm{Ni}_{50} \mathrm{Mn}_{35.5} \operatorname{In}_{14.5}$ for three orientations of sample - rotation around the $x$ - (a), $y$ - (b), $z$-axis (c).

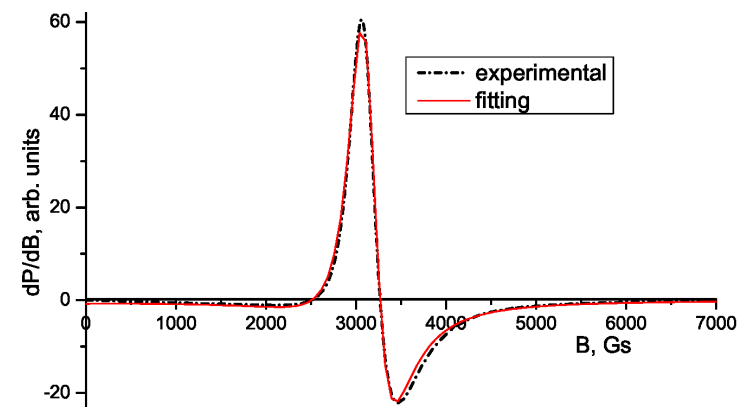

Fig. 4. The sample EMR line of $\mathrm{Ni}_{50} \mathrm{Mn}_{35.5} \operatorname{In}_{14.5}$ fitted by derivative of the Dyson function.

$$
g_{\text {eff }}=\frac{h \nu}{\mu_{\mathrm{B}} B_{\text {res }}},
$$

where $h$ is the Planck constant, $\nu$ is microwave frequency and $\mu_{\mathrm{B}}-$ Bohr magneton. The angular dependences of $B_{\text {res }}$ allow to find values of coefficients of effective $g$ tensor 


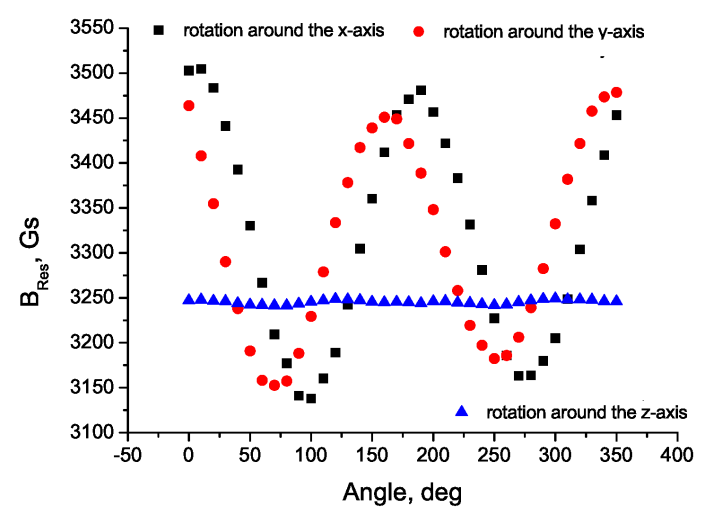

Fig. 5. The angular dependence of resonance field $B_{\text {res }}$ of $\mathrm{Ni}_{50} \mathrm{Mn}_{35.5} \mathrm{In}_{14.5}$ ribbon for three orientations of sample.

$$
\hat{g}=\left[\begin{array}{ccc}
2.0829(15) & -0.0014(15) & 0.0783(22) \\
& 2.0774(14) & 0.0946(22) \\
& & 1.9932(17)
\end{array}\right] .
$$

The calculation was done with the use of a computer program EPR-NMR (version 6.5v) [12]. The numerical procedure implemented in it allows to fit angular dependences of signal to the main $g$ ellipsoid axes $x^{\prime}, y^{\prime}, z^{\prime}$ and their orientations in relation to the labolatory axes $x, y$, $z$ (Fig. 6). As a result we obtain the principal values of $g_{e f f}$ :

$$
g_{x x}=2.1657(23), g_{y y}=2.0821(18), g_{z z}=1.9057(24)
$$

and angles between two mentioned refence systems (see Table I).

\section{TABLE I}

Polar coordinates of $x^{\prime} y^{\prime} z^{\prime}$ system in relation to labolatory system $x y z$.

\begin{tabular}{c|c|c|c}
\hline \hline & $x^{\prime}$ & $y^{\prime}$ & $z^{\prime}$ \\
\hline$\theta$ & $125.43(30)$ & $88.83(55)$ & $35.46(31)$ \\
$\phi$ & $228.6(13)$ & $139.5(11)$ & $231.1(11)$
\end{tabular}

\section{Conclusions}

The $\mathrm{Ni}_{50} \mathrm{Mn}_{35.5} \operatorname{In}_{14.5}$ Heusler alloys ribbons were investigated by EMR spectroscopy. Angular dependence of EMR was registered at $300 \mathrm{~K}$ for three perpendicular orientations of the sample. The obtained EMR spectra were correctly fitted by the Dyson function. Single, asymmetric line exhibits a strong angular dependence and magnetic anisotropy. The angular dependence of signals was used to determine the principal values of $g_{e f f}$.

\section{Acknowledgments}

The experiment was done in the laboratory 1.4 in the Center for Innovation and Transfer of Natural Sciences and Engineering Knowledge of the University of Rzeszów.

Computer Program EPR-NMR (Department of Chemistry, University of Saskatchewan, Saskatoon, Canada) was used for calculation.

\section{References}

[1] T. Krenke, E. Duman, M. Acet, E.F. Wassermann, X. Moya, L. Mañosa, A. Planes, Nat. Mater. 4, 450 (2005).

[2] Y. Suoto, Y. Imano, N. Koeda, T. Omori, R. Kainuma, K. Ishida, K. Oikawa, Appl. Phys. Lett. 85, 4358 (2004).

[3] Chun-Mei Li, Hu-Bin Luo, Qing-Miao Hu, Rui Yang, B. Johansson, L. Vitoset, Phys. Rev. B 86, 214205 (2012).

[4] S. Mizukami, A.A. Serga, J. Phys. D. Appl. Phys. 48, 160301 (2015).

[5] Zhe Li, Chao Jing, Jiping Chen, Shujuan Yuan, Shixun Cao, Jincang Zhang, Appl. Phys. Lett. 91, 112505 (2007).

[6] T. Kanomata, T. Yasuda, S. Sasaki, H. Nishihara, R. Kainuma, W. Ito, K. Oikawa, K. Ishida, K.U. Neumann, K.R.A. Ziebeck, J. Magn. Magn. Mater. 321, 773 (2009).

[7] R.A. de Groot, F.M. Mueller, P.G. van Engen, K.H.J. Buschow, Phys. Rev. Lett. 50, 2024 (1983).

[8] W. Maziarz, Solid State Phenom. 186, 251 (2012).

[9] F.J. Dyson, Phys. Rev. 98, 349 (1955).

[10] J.P. Joshi, S.V. Bhat, J. Magn. Reson. 168, 284 (2004).

[11] J.A. Weil, J.R. Bolton, Electron Paramagnetic Resonance: Elementary Theory and Practical Applications, 2nd ed., Wiley 2007.

[12] "EPR-NMR" v. 6.5, Department of Chemistry, University of Saskatchewan, Saskatoon, Canada. 